\title{
ALMOST MIZOHATA OPERATORS ${ }^{1}$ \\ BY \\ NICHOLAS HANGES
}

ABSTRACT. The range of every almost Mizohata operator is characterized via an integral formula.

Introduction. Let $(x, t)$ be coordinates in $R^{2}$. One of the simplest examples of a nonsolvable operator is the Mizohata operator

$$
M=\partial / \partial t+2 i t \partial / \partial x
$$

In fact if $f$ is a smooth function defined near the origin, there exists a distribution $u$ such that

$$
M u=f \quad \text { near the origin }
$$

if and only if $N f$ is analytic near zero. $N$ is defined by

$$
(N f)(x)=\int_{-\infty}^{\infty} \int_{-\infty}^{\infty} \int_{0}^{\infty} e^{i\left[x-y+i s^{2}\right] \xi} \frac{d \xi}{2 \pi} f(y, s) d y d s
$$

Recall that a similar result [4] holds for the celebrated Hans Lewy operator, where the role of $N$ is played by the Szegö operator.

A famous result of Nirenberg [6] shows that one may perturb $M$ so that the resulting operator has only the constant functions as solutions to the homogeneous equation. Later Treves [8, 10] and Sjöstrand [7] studied the class of almost Mizohata operators (defined in $\$ 1$ here). This class includes the Nirenberg example mentioned above. These authors obtained results on the homogeneous equation.

Our goal here is to characterize the range of all almost Mizohata operators via a formula analogous to (0.3). This is Theorem 3.1, stated at the end of the paper. In order to make this article self-contained we have included results of Treves [8] and Sjöstrand [7].

In $\$ 1$ the main result is Proposition 1.3 which gives a normal form for any almost Mizohata operator. In $\$ 2$ we characterize all solutions to the homogeneous equation. Although the results in these two sections have appeared in $[7,8,10]$, we believe this approach is necessary to understand Theorem 3.1 .

Received by the editors March 7, 1985.

1980 Mathematics Subject Classification. Primary 35C15; Secondary 35F05.

${ }^{1}$ This work has been partially supported by an Alfred P. Sloan Fellowship, a visiting membership at Courant Institute and Grants MCS8300553, MCS-82-01599, ARO-DAAG29-81-K-0043. 
Those familiar with hypoanalytic structures $[2,5]$ will observe that each almost Mizohata operator induces on the real line two natural hypoanalytic structures. Most results obtained here could have been stated in terms of the two corresponding hypoanalytic wave front sets. To keep the paper self-contained, we have avoided explicit mention of hypoanalyticity.

At this point we would like to thank François Treves for sharing the manuscript [10] with us.

1. Almost Mizohata operators and the normal form. Let $L$ be a smooth complex valued vector field defined near a point $p \in R^{2}$. Let $\bar{L}$ be its complex conjugate and $[L, \bar{L}]$ the commutator. We say that $L$ is an almost Mizohata operator near $p$ if: $L(p)$ and $\bar{L}(p)$ are linearly dependent,

Our objective in this section is to obtain a normal form for such operators. This is described in Proposition 1.3. We begin with some preliminary remarks.

After a smooth change of coordinates rectifying the real (or imaginary) part of $L$ and division by a nonvanishing smooth factor we may assume that there exist local coordinates $(x, t)$ defined near the origin in $R^{2}$ such that $L$ takes the form

$$
L=\frac{\partial}{\partial t}+i \lambda(x, t) \frac{\partial}{\partial x},
$$

where $\lambda$ is smooth and real valued. Since (1.1) and (1.2) are invariant conditions we have

$$
\begin{gathered}
\lambda(0,0)=0, \\
\frac{\partial \lambda}{\partial t}(0,0) \neq 0 .
\end{gathered}
$$

We will make one more preliminary observation. Let $u(x, t)$ be a smooth real function defined near the origin satisfying there the following:

$$
\lambda_{t} u_{t}+\lambda^{2} \lambda_{x} u_{x}=0, \quad u_{x}(0,0) \neq 0, \quad u(0,0)=0 .
$$

Now if we let $\tilde{x}=u(x, t)$ and $\tilde{t}=\lambda(x, t)$, it is a straightforward computation to check that the correspondence $(x, t) \mapsto(\tilde{x}, \tilde{t})$ is a smooth change of coordinates from a neighborhood of the origin into itself. Furthermore in these coordinates $L$ is transformed into

$$
\tilde{L}=\frac{\partial}{\partial \tilde{t}}+2 i \tilde{t} \tilde{\lambda}(\tilde{x}, \tilde{t}) \frac{\partial}{\partial \tilde{x}},
$$

where $\tilde{\lambda}$ is smooth, real and $\tilde{\lambda}(0,0) \neq 0$.

Throughout this article a function will be called flat at $\{t=0\}$ if it and all its derivatives vanish on the line $\{t=0\}$.

Lemma 1.1. Let $L$ be an almost Mizohata operator near $p \in R^{2}$. Then there exists a smooth diffeomorphism mapping a neighborhood of $p$ to a neighborhood of the origin so that in the new coordinates $(x, t)$ we have

$$
L=\frac{\partial}{\partial t}+2 i t(1+\rho(x, t)) \frac{\partial}{\partial x},
$$

where $\rho$ is smooth, real valued and flat at $\{t=0\}$. 
Proof. Our preliminary remarks allow us to assume that $L$ is defined near the origin and has the form

$$
L=\frac{\partial}{\partial t}+2 i t \lambda(x, t) \frac{\partial}{\partial x}
$$

with $\lambda$ real, smooth and $\lambda(0,0)>0$. We begin by formally solving the initial value problem

$$
\left\{\begin{array}{l}
L v=0 \\
\left.v\right|_{t=0}=x
\end{array}\right.
$$

The formal Taylor series of $\lambda$ determines the formal Taylor series for $v$ :

$$
v(x, t) \sim x+u_{1}(x) t+u_{2}(x) t^{2}+\cdots .
$$

Now define

$$
u(x, t)=x+u_{1}(x) t+u_{2}(x) t^{2}+\sum_{j=3}^{\infty} u_{j}(x) t^{j} \chi\left(\frac{t}{\varepsilon_{j}}\right)
$$

where $\chi \in C^{\infty}(\mathbf{R}), \chi(t)=1$ for $|t| \leqslant 1, \chi(t)=0$ for $|t| \geqslant 2$ and where the $\varepsilon_{j} \rightarrow 0$ are determined so that the series converges in $C^{\infty}$.

Note that $L u$ is flat at $\{t=0\}$. This implies that $u_{1}=0$ and $u_{2}(x)=-i \lambda(x, 0)$. Hence $(\operatorname{Re} u)(x, t)=x+O\left(t^{3}\right)$ and $(\operatorname{Im} u)(x, t)=-i \lambda(x, 0) t^{2}+O\left(t^{3}\right)$. Now define $\tilde{x}=\operatorname{Re} u, \tilde{t}=t \sqrt{-\operatorname{Im} u / t^{2}}$. This is clearly a smooth change of coordinates from a neighborhood of the origin to itself. $L$ now becomes

$$
\tilde{L}=\frac{\partial}{\partial \tilde{t}}+2 i \tilde{t}(1+\tilde{\rho}(x, t)) \frac{\partial}{\partial \tilde{x}},
$$

where $\tilde{\rho}$ is smooth, flat at $\{t=0\}$ but not necessarily real valued. To complete the proof we observe that there is a standard change of coordinates rectifying the real part of $L$ which also takes $L$ into the desired form (1.6). The proof is complete.

From now on, if $U$ is a neighborhood of the origin we denote by $U^{-}$the set

$$
U^{-}=\{(x, t) \in U: t<0\}
$$

and by $\bar{U}^{-}$its closure. A similar definition holds for $U^{+}$.

LEMMA 1.2. Let $L$ be defined near the origin having the form (1.6). Then there exists a neighborhood of the origin $U$ and $Z \in C^{\infty}\left(\bar{U}^{-}\right)$such that $L Z=0$ in $U^{-}, \partial Z / \partial x \neq 0$ in $\bar{U}^{-}, \operatorname{Im} Z \leqslant 0$ in $\bar{U}^{-}$and $\left.Z\right|_{t=0}$ is real valued.

Proof. Define $Y=-t^{2}$ and $X=x$. Then the map $(x, t) \rightarrow(X, Y)$ is a smooth change of coordinates from $U^{-}$into the lower half of the complex plane. In the new coordinates we have

$$
L=2 \sqrt{-Y} E
$$

where $E$ is defined by

$$
E=\frac{\partial}{\partial Y}-i(1+\rho(X,-\sqrt{-Y})) \frac{\partial}{\partial X}
$$


for $Y<0$. Note that $E$ is elliptic for $Y<0$ small and $\rho(X,-\sqrt{-Y})$ is smooth up to $\{Y=0\}$ and flat there. Next define $\tilde{\rho}$ by

$$
\tilde{\rho}(X, Y)= \begin{cases}0, & Y \geqslant 0 \\ \rho(X,-\sqrt{-Y}), & Y<0\end{cases}
$$

and define $\tilde{E}$ by

$$
\tilde{E}=\frac{\partial}{\partial Y}-i(1+\tilde{\rho}(X, Y)) \frac{\partial}{\partial X} .
$$

Observe that $\tilde{E}$ is elliptic in a neighborhood of the origin and has smooth coefficients. Hence, it is known (see for example [3]) that there exists a smooth function $\tilde{W}(X, Y)$ defined near the origin such that $\tilde{E} \tilde{W}=0$ near the origin and $(\partial \tilde{W} / \partial X)(0,0) \neq 0$. Letting $W(x, t)=\tilde{W}\left(x,-t^{2}\right)$ for $t<0$ we see that there is a neighborhood of the origin $V$ such that

$$
L W=0 \quad \text { in } V^{-}
$$

with $W \in C^{\infty}\left(\bar{V}^{-}\right)$and $\partial W / \partial X \neq 0$ in $\bar{V}^{-}$.

Now let $W=a+i b$ where $a, b$ are real. We may assume that $\partial a / \partial x>0$ and $W(0,0)=0$. Define $b_{0}(x)=b(x, 0), a_{0}(x)=a(x, 0)$ and $a_{0}^{-1}(x)$ to be the local inverse of $a_{0}$. Next define $F(x)=b_{0}\left(a_{0}^{-1}(x)\right)$. It follows directly from (1.4) and the fact that $\partial a / \partial x \neq 0$ that (perhaps after shrinking $V$ ) we have

$$
F(a(x, t)) \geqslant b(x, t), \quad(x, t) \in \bar{V}^{-} .
$$

Hence we see that

$$
W: \bar{V}^{-} \rightarrow\{X+i Y \in \mathbf{C} \mid Y \leqslant F(X)\}=\mathcal{O} .
$$

If $V$ is small enough, $\mathcal{O}$ will be simply connected. Hence by the Riemann mapping theorem we can find $H$ holomorphic in the interior of $\mathcal{O}$ and smooth up to $\{Y=F(X)\}$ mapping the interior of $\mathcal{O}$ conformally into the lower half disk

$$
\left\{u+i v \in \mathbf{C} \mid u^{2}+v^{2}<1, v<0\right\} \text {. }
$$

$H$ also can be chosen to map the curve $Y=F(X)$ to the real axis. Now if we define

$$
Z(x, t)=H(W(x, t)),
$$

we see that $Z$ has all the desired properties. Q.E.D.

We are now ready to state the main result of this section:

Proposition 1.3. Let $L$ be an almost Mizohata operator near $p \in R^{2}$. Then there exists a smooth diffeomorphism mapping a neighborhood of $p$ to a neighborhood of the origin $U$, so that in the new coordinates $(x, t)$ we have

$$
L=\frac{\partial}{\partial t}+2 i t(1+\rho(x, t)) \frac{\partial}{\partial x},
$$

where $\rho$ is smooth, real valued and $\rho(x, t)=0$ for $(x, t) \in U^{-}$.

Proof. We may assume that there exists a neighborhood of the origin $U$ so that $L$ has the form (1.6) and that there exists a function $Z: \bar{U}^{-} \rightarrow \mathbf{C}$ as described in Lemma 1.2. Let $Z=a+i b$ with $a, b$ real. Assume $\partial a / \partial x \neq 0, Z(0,0)=0$. Since 
$L Z=0$ in $U^{-}$and $b(x, 0)=0$ we see that

$$
b(x, t)=-t^{2} v(x, t), \quad(x, t) \in \bar{U}^{-},
$$

where $v(x, t)>0,(x, t) \in \bar{U}^{-}$. Now define

$$
\begin{gathered}
\tilde{t}=\left\{\begin{array}{lc}
t \sqrt{v(x,-t)}, & t>0, \\
t \sqrt{v(x, t)}, & t \leqslant 0,
\end{array}\right. \\
\tilde{x}=\left\{\begin{array}{lc}
a(x,-t), & t>0, \\
a(x, t), & t \leqslant 0 .
\end{array}\right.
\end{gathered}
$$

The map $(x, t) \mapsto(\tilde{x}, \tilde{t})$ is smooth. Indeed, $L Z=0$ in $U^{-}$implies that

$$
\frac{\partial^{2 j+1} Z}{\partial t^{2 j+1}}(x, 0)=0, \quad j=0,1,2, \ldots
$$

Furthermore, it is easy to check that the above map is a change of coordinates. Now a straightforward calculation shows that $L$ has the required form in the $(\tilde{x}, \tilde{t})$ coordinates, modulo a smooth nonvanishing factor.

REMARK 1.4. Suppose that $L$ has the form (1.6) near the origin. Suppose further that there exists a smooth $u$ such tha: $L u=0$ and $\partial u / \partial x \neq 0$ near the origin. Then an argument similar to that just given shows that there is a smooth coordinate change taking $L$ into a nonvanishing multiple of the Mizohata operator $\partial / \partial t+$ $2 i t \partial / \partial x$.

2. The homogeneous equation. Let $L$ be an almost Mizohata operator near $p \in R^{2}$. Our aim in this section is to study the local structure of distributions $u$ such that

$$
L u=0 .
$$

Note that our approach is greatly inspired by [1]. Throughout this section we will assume that $L$ is defined in a neighborhood $U$ of the origin and that $L$ has the form (1.19).

We begin by observing that there exists a smooth function $Z \in C^{\infty}\left(\bar{U}^{+}\right)$such that $Z(0,0)=0, L Z=0$ in $U^{+}, \partial Z / \partial X \neq 0$ in $\bar{U}^{+}, \operatorname{Im} Z \leqslant 0$ in $\bar{U}^{+}$, and $\left.Z\right|_{t=0}$ is real valued. The existence of such a $Z$ follows from arguments similar to those in the proof of Lemma 1.2. Note that the function $x-i t^{2}$ in $U^{-}$is the analog of $Z$. Distributions $u$ satisfying (2.1) will then be described in terms of $Z$ and $x-i t^{2}$. We pause now to recall a definition.

A function $H$ is said to be holomorphic of slow growth in the lower half plane if and only if there exists $U$, a neighborhood of the origin in the complex plane, such that $H$ is holomorphic in $U^{-}$and there exists $C>0$ and an integer $N \geqslant 0$ such that

$$
|H(x+i y)| \leqslant C /|y|^{N}, \quad x+i y \in U^{-} .
$$

Note that it is a simple exercise to show that $H$ is holomorphic of slow growth in the lower half plane if and only if there exists a neighborhood $U$ of the origin in the complex plane and $J$ holomorphic in $U^{-}$, continuous up to the real axis and an integer $k \geqslant 0$ such that

$$
H=\partial^{k} J / \partial Z^{k} \quad \text { in } U^{-} \text {. }
$$


THEOREM 2.1. Let $L$ be defined in a neighborhood of the origin having the form (1.19) and let $Z$ be as defined above. Assume that there exists a distribution defined near the origin such that $L u=0$ there. Then there exists a neighborhood of the origin $U$ and two functions $H$ and $J$, holomorphic of slow growth in the lower half plane such that

$$
u(x, t)= \begin{cases}H(Z(x, t)), & (x, t) \in U^{+}, \\ J\left(x-i t^{2}\right), & (x, t) \in U^{-} .\end{cases}
$$

REMARK 2.2. Since the lines $\{t=$ constant $\}$ are noncharacteristic for $L$, we may view $u$ as a smooth function in $t$ with values in the distributions in $x$. In particular the trace $u_{0}(x)=u(x, 0)$ is a distribution in one variable, well defined near the origin. Theorem 2.1 then implies that

$$
\lim _{t \rightarrow 0^{+}} H(Z(x, t))=u_{0}(x)=\lim _{t \rightarrow 0^{-}} J\left(x-i t^{2}\right),
$$

where the limits are taken in the distribution sense. Furthermore, it is easy to see that condition (2.5) is sufficient to locally solve the Cauchy problem

$$
L u=0, \quad u(x, 0)=u_{0}(x) .
$$

Note that condition (2.5) may be very strong. Indeed for the Nirenberg example mentioned in the introduction, the only $u_{0}$ satisfying (2.5) are the constant functions.

Proof of Theorem 2.1. Let $u$ be a distribution defined near the origin such that $L u=0$. The preceding remarks allow us to define the distribution

$$
\tilde{u}(x, t)= \begin{cases}u(x, t), & t \geqslant 0, \\ u(x,-t), & t<0 .\end{cases}
$$

Also define

$$
\tilde{Z}(x, t)= \begin{cases}Z(x, t), & t \geqslant 0 \\ Z(x,-t), & t<0 .\end{cases}
$$

Note that $\tilde{Z}$ is smooth since $\left(\partial^{2 k+1} Z / \partial t^{2 k+1}\right)(x, 0)=0$ for $k=0,1,2, \ldots$ Also define

$$
\tilde{\lambda}(x, t)= \begin{cases}2 i t(1+\rho(x, t)), & t \geqslant 0, \\ 2 i t(1+\rho(x,-t)), & t<0,\end{cases}
$$

which is smooth since $\rho$ is flat as $\{t=0\}$. Now define

$$
\tilde{L}=\frac{\partial}{\partial t}+\tilde{\lambda}(x, t) \frac{\partial}{\partial x}
$$

and observe that

$$
\tilde{L} \tilde{Z}=0=\tilde{L} \tilde{u} .
$$

Define $\tilde{L}_{0}=\left(1 / \tilde{Z}_{x}\right) \partial / \partial x$. Note that we have

$$
\left[\tilde{L}_{0}, \tilde{L}\right]=0 \text {. }
$$


Since $\tilde{u}$ is locally of finite order there exists an integer $N$ and a continuous function $v$ defined near the origin such that

$$
\tilde{L}_{0}^{N} v=\tilde{u} .
$$

It follows from (2.11) and (2.12) that

$$
\tilde{L} v=\sum_{j=0}^{N-1} c_{j}(t) Z(x, t)^{j},
$$

where the $c_{j}$ are distributions in $t$ only. Let $d_{j}, j=0, \ldots, N-1$, be distributions in $t$ only such that $\partial d_{j} / \partial t=c_{j}, j=0, \ldots, N-1$, and define

$$
w(x, t)=\sum_{j=0}^{N-1} d_{j}(t) Z(x, t)^{j} .
$$

Hence $\tilde{L}(v-w)=0$, with $v-w$ smooth in $t$ with values in the distributions in $x$. Since $v$ is continuous, a simple induction on $N$ shows that $v-w$ is also continuous. Now for $t>0$, the map $(x, t) \rightarrow Z(x, t)$ is a diffeomorphism into the lower half plane taking $L$ into a multiple of $\partial / \partial \bar{Z}$. Hence we see that there is a holomorphic function $J$ in the lower half plane such that

$$
v-w=J \circ Z \text {. }
$$

Since $v-w$ is continuous, $J$ is continuous up to the real axis. Hence for $t>0$ we have

$$
u=\tilde{u}=\tilde{L}_{0}^{N} v=\tilde{L}_{0}^{N}(J \circ Z+w)=\tilde{L}_{0}^{N}(J \circ Z)=\left(\partial^{N} J / \partial Z^{N}\right) \circ Z .
$$

Since $J$ is continuous to the real axis, it follows that $\partial^{N} J / \partial Z^{N}$ is holomorphic of slow growth in the lower half plane. Similar arguments apply for $t<0$. Q.E.D.

The next result shows that the equation $L u=0$ will have many solutions provided that the trace $Z_{0}(x)=Z(x, 0)$ is very well behaved.

THEOREM 2.3. Let $L$ be defined in a neighborhood of the origin having the form (1.19) and $Z$ be as defined above. Then there exists a neighborhood of the origin $U$ and $u \in C^{\infty}(U)$ such that

$$
L u=0 \quad \text { in } U, \quad \partial u / \partial x \neq 0 \quad \text { in } U
$$

if and only if $Z_{0}$ is real analytic near the origin.

REMARK 2.4. Note that Theorem 2.3 combined with Remark 1.4 shows that the local analyticity of $Z_{0}$ is equivalent to the fact that $L$ can be transformed into the Mizohata operator.

Proof of Theorem 2.3. Let $U$ be a neighborhood of the origin and $u \in C^{\infty}(U)$ such that (2.17) holds. We may assume that the trace $u(x, 0)$ is real valued. Indeed this follows from an application of the Riemann mapping theorem, as in the proof of Lemma 1.2. By Theorem 2.1 we see that there exists a function $J$ holomorphic in the lower half plane such that

$$
u(x, t)=J\left(x-i t^{2}\right), \quad(x, t) \in U^{-} .
$$


Our assumptions on $u$ show that $J$ is real on the real axis and smooth up to the real axis. Hence the trace $u(x, 0)$ is real analytic near the origin by the reflection principle. Similarly there exists $H$ holomorphic in the lower half plane such that

$$
u(x, t)=H(Z(x, t)), \quad(x, t) \in \bar{U}^{+} .
$$

Again $H$ is smooth up to the real axis and real valued there. Hence the trace of $H$ on the real axis is real analytic. Furthermore, $\partial H / \partial x \neq 0$. Hence $Z_{0}$ is real analytic near the origin.

Next assume that $Z_{0}$ is real analytic near the origin. For small $(x, t)$ define

$$
u(x, t)= \begin{cases}Z(x, t), & t \geqslant 0 \\ Z_{0}\left(x-i t^{2}\right), & t<0 .\end{cases}
$$

It follows that $u$ is smooth since $\left(\partial^{2 k+1} Z / \partial t^{2 k+1}\right)(x, 0)=0$ for $k=0,1,2, \ldots$ Q.E.D.

3. Characterization of the range. Let $L$ be an almost Mizohata operator defined in a neighborhood of the origin $U$ having the form (1.19). Our aim in this section is to characterize all smooth $f$ defined near the origin for which there exists a distribution $u$ defined near the origin such that

$$
L u=f .
$$

Throughout this section we will use the function $Z$ introduced in $\S 2$. In addition to the properties of $Z$ listed there, we may also assume that $(\partial Z / \partial x)(0,0)=1$.

Let $f \in C^{\infty}(U)$. We will begin by trying to construct a solution $u$ to (3.1) as a Fourier integral distribution. In general no such $u$ need exist since $L$ is not solvable near the origin. But, in the course of our attempt, a condition on $f$ will naturally appear. This will allow us to solve (3.1). Later we will show this condition to be also necessary for (3.1) to hold.

Before starting we pause to recall a generalized Fourier inversion formula. Let $g \in C_{0}^{\infty}\left(U^{+}\right)$and suppose we know that

$$
|\operatorname{Im}(Z(x, t)-Z(y, t))| \leqslant \frac{1}{2}|\operatorname{Re}(Z(x, t)-Z(y, t))|
$$

for $(x, t) \in U^{+},(y, t) \in U^{+}$. Then we have

$$
g(x, t)=\lim _{\varepsilon \rightarrow 0^{+}} \int_{-\infty}^{\infty} \int_{-\infty}^{\infty} e^{i[Z(x, t)-Z(y, t)] \xi-\varepsilon \xi^{2}} g(y, t) d Z(y, t) \frac{d \xi}{2 \pi} .
$$

Here the convergence is valid in $C^{\infty}\left(U^{+}\right)$. If $g \in C_{0}^{k}\left(U^{+}\right)$or $\mathrm{E}^{\prime}\left(U^{+}\right)$, then (3.3) is valid in these topologies also. For a proof see [2, p. 344]. We are now ready to attempt to solve (3.1). First choose $\chi \in C_{0}^{\infty}(U)$ such that $\chi=1$ near the origin, with supp $\chi$ contained in a neighborhood where $f$ is defined. We introduce

$$
u^{22}(x, t)=-\lim _{\varepsilon \rightarrow 0^{+}} \int_{t}^{\infty} \int_{-\infty}^{\infty} \int_{0}^{\infty} e^{i[Z(x, t)-Z(y, s)] \xi-\varepsilon \xi^{2}} \frac{d \xi}{2 \pi}(\chi f)(y, s) d Z(y, s) d s .
$$

We claim that if $U$ is chosen small enough, $u^{22} \in C^{\infty}\left(\bar{U}^{+}\right)$. This will take several steps to prove. 
Write $Z=a+i b, a$ and $b$ real. $L Z=0$ is then equivalent to

$$
\begin{gathered}
a_{t}=2 t(1+\rho) b_{x}, \\
b_{t}=-2 t(1+\rho) a_{x} .
\end{gathered}
$$

Since $a_{x}(0,0)=1$, we see by integrating (3.6) with respect to $t$ that, if $U$ is small enough, there exists $C_{1}>0$ such that

$$
b(x, t)-b(x, s) \geqslant C_{1}\left(s^{2}-t^{2}\right)
$$

for $(x, t) \in \bar{U}^{+},(x, s) \in \bar{U}^{+}$.

Since $b_{x}(0,0)=0$, by integrating (3.5) with respect to $t$ we see that if $U$ is small enough we have

$$
|a(x, t)-a(x, s)| \leqslant C_{1}\left|t^{2}-s^{2}\right|
$$

for $(x, t) \in \bar{U}^{+},(x, s) \in \bar{U}^{+}$. The fact that $b_{x}(0,0)=0$ also implies that if we shrink $U$ we may assume

$$
|b(x, t)-b(y, t)| \leqslant \frac{1}{8}|x-y|
$$

for $(x, t) \in \bar{U}^{+},(y, t) \in \bar{U}^{+}$.

Now (3.5) and that fact that $a_{x}(0,0)=1$ imply that

$$
a(x, t)=x+O\left(x^{2}\right)+O\left(t^{2}\right) .
$$

Hence we have, if $U$ is small, a smooth $A$ such that

$$
a(x, t)-a(y, t)=x-y+A(x, y, t) \text { in } \bar{U}^{+}
$$

with $|A(x, y, t)| \leqslant \frac{1}{4}|x-y|$.

The first problem in studying (3.4) is that of estimating the exponential term. We see that we may deform the path of $\xi$ integration into the contour (as in $[9, \S 6]$ )

$$
\zeta=\rho\left(1+\frac{i}{2} \frac{x-y}{|x-y|}\right), \quad \rho>0,
$$

in the complex plane.

We must now estimate, assuming $0 \leqslant t \leqslant s$,

$$
\begin{aligned}
\operatorname{Im}[(Z & \left.(x, t)-Z(y, s)) \zeta+i \varepsilon \zeta^{2}\right] \\
\quad & \rho(b(x, t)-b(y, s))+\frac{\rho}{2} \frac{(x-y)}{|x-y|}(a(x, t)-a(y, s))+\frac{3}{4} \varepsilon \rho^{2} \\
\geqslant & \rho C_{1}\left(s^{2}-t^{2}\right)-\frac{1}{8} \rho|x-y|-\frac{\rho}{2} C_{1}\left|t^{2}-s^{2}\right| \\
& +\frac{\rho}{2}|x-y|-\frac{1}{8} \rho|x-y|+\frac{3}{4} \varepsilon \rho^{2} \\
\geqslant & \frac{\rho}{2} C_{1}\left(s^{2}-t^{2}\right)+\frac{\rho}{2}|x-y|+\frac{3}{4} \varepsilon \rho^{2} \geqslant 0
\end{aligned}
$$

for $(x, t) \in \bar{U}^{+},(y, s) \in \bar{U}^{+}$. The above estimates follow from (3.7), (3.8), (3.9) and (3.11). 
Next we introduce the operator

$$
L_{0}=\frac{1}{Z_{x}} \frac{\partial}{\partial x}
$$

and its formal transpose $L_{0}^{\prime}$. It is easy to show by induction that for any integer $N \geqslant 1$ we have

$$
\left(1-L_{0}\right)^{N}\left(e^{-i Z \xi}\right)=(1+i \xi)^{N}\left(e^{-i Z \xi}\right)
$$

and

$$
\left(1-L_{0}^{\prime}\right)^{N}\left(Z_{x} v\right)=Z_{x}\left(1+L_{0}\right)^{N} v
$$

for any smooth $v$.

Hence we see that the right side of (3.4) can be written as

$$
\begin{gathered}
-\int_{t}^{\infty} \int_{-\infty}^{\infty} \int_{0}^{\infty} e^{i Z(x, t) \xi-\varepsilon \xi^{2}}(1+i \xi)^{-N}\left(1-L_{0}\right)^{N} \\
\cdot\left(e^{-i Z(y, s) \xi}\right) \frac{d \xi}{2 \pi}(\chi f)(y, s) d Z(y, s) d s \\
=-\int_{t}^{\infty} \int_{-\infty}^{\infty} \int_{0}^{\infty} e^{i[Z(x, t)-Z(y, s)] \xi-\varepsilon \xi^{2}} \\
\cdot(1+i \xi)^{-N} \frac{d \xi}{2 \pi}\left(1+L_{0}\right)^{N}(\chi f)(y, s) d Z(y, s) d s .
\end{gathered}
$$

Since we can choose $N$ as large as we please in (3.18) and we can make the deformation (3.12), it follows that $u^{22} \in C^{\infty}\left(\bar{U}^{+}\right)$.

Using similar arguments we also define

$$
u^{21}(x, t)=\lim _{\varepsilon \rightarrow 0^{+}} \int_{0}^{t} \int_{-\infty}^{\infty} \int_{-\infty}^{0} e^{i[Z(x, t)-Z(y, s)] \xi-\varepsilon \xi^{2}} \frac{d \xi}{2 \pi}(\chi f)(y, s) d Z(y, s) d s
$$

for $(x, t) \in \bar{U}^{+}$,

$$
u^{11}(x, t)=\lim _{\varepsilon \rightarrow 0^{+}} \int_{0}^{t} \int_{-\infty}^{\infty} \int_{-\infty}^{0} e^{i\left[x-y+i\left(s^{2}-t^{2}\right)\right] \xi-\varepsilon \xi^{2}} \frac{d \xi}{2 \pi}(\chi f)(y, s) d y d s
$$

for $(x, t) \in \bar{U}^{-}$.

$$
u^{12}(x, t)=\lim _{\varepsilon \rightarrow 0^{+}} \int_{-\infty}^{t} \int_{-\infty}^{\infty} \int_{0}^{\infty} e^{i\left[x-y+i\left(s^{2}-t^{2}\right)\right] \xi-\varepsilon \xi^{2}} \frac{d \xi}{2 \pi}(\chi f)(y, s) d y d s
$$

for $(x, t) \in \bar{U}^{-}$.

We see that $u^{21}, u^{22} \in C^{\infty}\left(\bar{U}^{+}\right)$and $u^{11}, u^{12} \in C^{\infty}\left(\bar{U}^{-}\right)$. Now let $H$ be the Heaviside function, that is

$$
H(t)= \begin{cases}1, & t \geqslant 0 \\ 0, & t<0\end{cases}
$$

Define $u$ as follows:

$$
u(x, t)=H(t)\left(u^{21}(x, t)+u^{22}(x, t)\right)+H(-t)\left(u^{11}(x, t)+u^{12}(x, t)\right) .
$$


Notice that (3.9) and (3.11) imply that (3.2) holds. Hence the Fourier inversion formula (3.3) is valid, and we have

$$
(L u)(x, t)=(\chi f)(x, t)-\delta(t) \otimes(K f)(x)
$$

for $(x, t) \in U$, where we define $K=K^{+}+K^{-}$,

$$
\left(K^{+} f\right)(x)=\lim _{\varepsilon \rightarrow 0^{+}} \int_{0}^{\infty} \int_{-\infty}^{\infty} \int_{0}^{\infty} e^{i[Z(x, 0)-Z(y, s)] \xi-\varepsilon \xi^{2}} \frac{d \xi}{2 \pi}(\chi f)(y, s) d Z(y, s) d s
$$

and

$$
\left(K^{-} f\right)(x)=\lim _{\varepsilon \rightarrow 0^{+}} \int_{-\infty}^{0} \int_{-\infty}^{\infty} \int_{0}^{\infty} e^{i\left(x-y+i s^{2}\right) \xi-\varepsilon \xi^{2}} \frac{d \xi}{2 \pi}(\chi f)(y, s) d y d s
$$

We now introduce the crucial condition:

There exist two holomorphic functions, $A$ and $B$, of slow growth in the lower half plane such that

$$
(K f)(x)=\lim _{t \rightarrow 0^{+}} A(Z(x, t))+\lim _{t \rightarrow 0^{-}} B\left(x-i t^{2}\right),
$$

where the limit is taken in the distribution sense.

If we assume that $f$ satisfies (3.27), we may define the distribution

$$
v(x, t)=H(t) A(Z(x, t))-H(-t) B\left(x-i t^{2}\right) .
$$

Note that

$$
(L v)(x, t)=\delta(t) \otimes(K f)(x) .
$$

Hence we see that

$$
L(u+v)=f \quad \text { near the origin. }
$$

So we see that (3.27) is sufficient to solve (3.1).

Assume that (3.1) holds for some smooth $f$. For the moment we will assume $u \in C^{1}$. The case of general distribution solutions will be handled later. Consider the quanity

$$
\left(K_{\varepsilon}^{+} f\right)(x)=\int_{0}^{\infty} \int_{-\infty}^{\infty} \int_{0}^{\infty} e^{i\left[Z(x, 0)-Z(y, s) \xi \xi-\varepsilon \xi^{2}\right.} \frac{d \xi}{2 \pi}(\chi f)(y, s) d Z(y, s) d s,
$$

where $\chi \in C_{0}^{\infty}\left(\mathbf{R}^{2}\right), \chi=1$ near the origin and the support of $\chi$ is contained in a neighborhood of 0 where both $u$ and $f$ are defined. Using the fact that (3.1) holds, we integrate by parts to obtain

$$
K_{\varepsilon}^{+} f=I_{\varepsilon}^{+} f+J_{\varepsilon}^{+} f
$$

where we define

$$
\left(I_{\varepsilon}^{+} f\right)(x)=-\int_{0}^{\infty} \int_{-\infty}^{\infty} \int_{0}^{\infty} e^{i[Z(x, 0)-Z(y, s)]-\varepsilon \xi^{2}} \frac{d \xi}{2 \pi}(L \chi)(y, s) u(y, s) d Z(y, s) d s
$$

and

$$
\left(J_{\varepsilon}^{+} f\right)(x)=-\int_{-\infty}^{\infty} \int_{0}^{\infty} e^{i[Z(x, 0)-Z(y, 0)] \xi-\varepsilon \xi^{\xi}} \frac{d \xi}{2 \pi} \chi(y, 0) u(y, 0) d Z(y, 0)
$$


First we analyse $I_{\varepsilon}^{+} f$. As before we pass to the contour (3.12). Estimate (3.13) then tells us that there exists $C_{1}>0$ such that

$$
\operatorname{Im}\left[(Z(x, 0)-Z(y, s)) \zeta+i \varepsilon \zeta^{2}\right] \geqslant \frac{\rho}{2} C_{1} s^{2}+\frac{\rho}{2}|x-y|+\frac{3}{4} \varepsilon \rho^{2} .
$$

Since $\chi=1$ near the origin we see that $L \chi=0$ near 0 . Hence either $y$ or $s$ is strictly bounded away from 0 throughout the domain of integration. It follows then that if $x$ is small enough, there exists $C>0$ so that

$$
\operatorname{Im}\left[(Z(x, 0)-Z(y, s)) \zeta+i \varepsilon \zeta^{2}\right] \geqslant C \rho+\frac{3}{4} \varepsilon \rho^{2} .
$$

This estimate implies that $\lim _{\varepsilon \rightarrow 0^{+}} I_{\varepsilon}^{+} f$ is a real analytic function of the variable $Z(x, 0)$. That is, there exists a function $A^{+}$holomorphic near the origin in $\mathbf{C}$ such that

$$
\lim _{\varepsilon \rightarrow 0^{+}}\left(I_{\varepsilon}^{+} f\right)(x)=A^{+}(Z(x, 0)) .
$$

Now we turn our attention to $J_{\varepsilon}^{+} f$. Observe that by the generalized Fourier inversion formula (3.3) we have for small $x$

$$
\begin{aligned}
\lim _{\varepsilon \rightarrow 0^{+}} J_{\varepsilon}^{+} f(x)= & -u(x, 0) \\
& +\lim _{\varepsilon \rightarrow 0^{+}} \int_{-\infty}^{\infty} \int_{-\infty}^{0} e^{i[Z(x, 0)-Z(y, 0)] \xi-\varepsilon \xi^{2}} \frac{d \xi}{2 \pi} \chi(y, 0) u(y, 0) d Z(y, 0) .
\end{aligned}
$$

In the last term of (3.37) we see that $\xi \leqslant 0$ throughout the domain of integration. Hence there exists a function $B^{+}$holomorphic of slow growth in the lower half plane such that

$$
\lim _{\varepsilon \rightarrow 0^{+}} J_{\varepsilon}^{+} f(x)=-u(x, 0)+\lim _{t \rightarrow 0^{+}} B^{+}(Z(x, t)) .
$$

Combining (3.36) and (3.38) we see that there exists a function $A$ holomorphic of slow growth in the lower half plane such that

$$
K^{+} f(x)=-u(x, 0)+\lim _{t \rightarrow 0^{+}} A(Z(x, t)) .
$$

Similar arguments also show that there exists a function $B$ holomorphic of slow growth in the lower half plane such that

$$
K^{-} f(x)=u(x, 0)+\lim _{t \rightarrow 0^{-}} B\left(x-i t^{2}\right) .
$$

Combining (3.39) and (3.40) we see that (3.27) is necessary for (3.1) to hold, assuming $u \in C^{1}$. In case (3.1) holds with $u \in \mathscr{D}^{\prime}$ we use the operator $L_{0}$ defined in (3.14). Since $u$ is locally of finite order we know there exists a $v \in C^{1}$ and an integer $N$ so that

$$
u=L_{0}^{N} v \quad \text { in } \bar{U}^{+} .
$$

Using the fact that $L$ and $L_{0}$ commute we may substitute $L_{0}^{N} v$ for $u$ in (3.30) and proceed in a straightforward way. We leave the details to the reader. We may summarize the results of this section in the following. 
THEOREM 3.1. Let L be an almost Mizohata operator defined in a neighborhood of the origin $U$ having the form (1.19). Let $f \in C^{\infty}(U)$. Then there exists a distribution $u$ such that $L u=f$ near the origin if and only if $f$ satisfies (3.27).

REMarK. Condition (3.27) is independent of the choice of the cut off function $\chi$.

\section{REFERENCES}

1. M. S. Baouendi and F. Treves, A local constancy principle for the solutions of certain overdetermined systems of first order linear PDE, Advances in Math., Suppl. Studies, Vol. 7A in honor of L. Schwartz, Academic Press, New York, 1981, pp. 245-262.

2. M. S. Baouendi, C. H. Chang and F. Treves, Microlocal hypo-analyticity and extension of $C R$ functions, J. Differential Geom. 18 (1983), 331-391.

3. R. Courant and D. Hilbert, Methods of mathematical physics, Vol. II, Interscience, New York, 1962.

4. P. C. Greiner, J. J. Kohn and E. M. Stein, Necessary and sufficient conditions for solvability of the Lewy equation, Proc. Nat. Acad. Sci. U.S.A. 72 (1975), 3287-3289.

5. N. Hanges and F. Treves, Propagation of holomorphic extendability of CR functions, Math. Ann. 263 (1983), 157-177.

6. L. Nirenberg, Lectures on linear partial differential equations, C.B.M.S. Regional Conf. Ser. in Math., no. 17, Amer. Math. Soc., Providence, R..I., 1973.

7. J. Sjöstrand, Note on a paper of F. Treves concerning Mizohata type operators, Duke Math. J. 47 (1980), 601-608.

8. F. Treves, Remarks about certain first order linear PDE in two variables, Comm. Partial Differential Equations 5 (1980), 381-425.

9. On the local solvability and local integrability of systems of vector fields, Acta Math. 151 (1983), 1-48.

10. Mizohata structures, unpublished

Department of Mathematics, lehman College (CUNy), Bedford Park Boulevard West, BRONX, NEW YORK 10468 